\title{
Integrated transport system - basis of Russia's security
}

\author{
Ruben Kazaryan ${ }^{1, *}$, Peraskovya Andreeva ${ }^{1}$, and Natalya Galaeva ${ }^{1}$ \\ ${ }^{1}$ Moscow State University of Civil Engineering, 129337, Yaroslavskoe shosse, 26, Moscow, Russia
}

\begin{abstract}
Possibility of improvement of optimization of integrated transport systems formed in case of several types of transport due to the introduction of design and construction management at the stage of creating subsystems of each type of transport. Factors intended to participate in the management of natural resources related to their extraction (technical and economic factors affecting the organization of movement by mode of transport and factors associated with the production of final transport products). The paper discusses the issues of placement and management capabilities of industrial transport systems based on the interaction of technical and natural systems. The implementation of project management methods for the construction of transport facilities developed on the basis of compiling individual project management algorithms, including organizational preparatory procedures, should be mandatory for all project participants, including customers.
\end{abstract}

\section{Introduction}

To date, the management of natural and technical systems have not been sufficiently developed. To assess the possibilities and conditions for the implementation of management in natural and technical systems, it is necessary to note their main properties:

- information ability and controllability - the ability to obtain information that allows forecasting the development of the system on the basis of its previous state; being subjected to a targeted influence of a person, the system goes into a state assigned to it.

- purposefulness - in the course of its operation, the system receives, transforms, accumulates, and uses information in accordance with the set goals.

- hierarchy - diversity of subsystems in the system, which form a complex structure with coordinated levels in the presence of standard indicators characterizing the system - the system and its components function under the influence of certain factors and processes (physical, energy, mechanical, etc.).

- integrity - the manifestation of functional system properties only as a result of interaction of individual subsystems in the system with the consistency of the goals of functioning - the functioning of individual subsystems must be carried out taking into account the uncompromising functioning of the entire system.

- sustainability - during operation, the system can move into various stable states, depending on disturbances from the environment;

\footnotetext{
*Corresponding author: r.kazarian@mail.ru
} 
- vitality - the ability of the system to function, even with the incomplete performance of its functions, after an unforeseen impact of destructive factors.

\section{Materials and Methods}

For detailed consideration of transport natural and technical systems, their classification is necessary, which, depending on the goals and objectives of the study, can be carried out in various directions. One of the main ones is the relating to geotechnical and environmental problems on the basis of the performed functions.

The main factors influencing the formation of a separate value and the role of the transport system within the natural and technical system of different types of transport can be divided into two large groups.

Both groups are related to land use.

1. Factors intended to participate in the management of natural resources associated with their extraction;

These include technical and economic factors affecting the organization of traffic by types of transport:

- composition and interposition of system objects;

- dynamics of technogenic load, volumes of waste entering the dumps and the aquatic environment, volumes of water use and water consumption;

- physical and chemical properties of discharged waste;

- heat load on water bodies, etc.

2. Factors intended to participate in the management of the natural environment in general, associated with the production of final products or semi-finished products.

This group includes physiographic factors that determine the possibility and conditions for the creation of a network of communication lines and affect mainly on land modes of transport:

- qualitative and quantitative indicators of the natural environment that have changed during the creation of engineering objects of the natural and technical system;

- structure and intensity of landscape connections;

- resource and reduction potential of the environment.

To assess the current state of land, the role and place of transport in territorial natural and technical systems, it is necessary to link to all categories of land in accordance with their intended purpose according to the Land Code of the Russian Federation. For designing the development of the transport network, data are required on the landscape and ecological features of the territory, the functional connections between their elements, as well as on the dynamic and static characteristics of the system. The boundaries of the geotechnical system are largely arbitrary and are directly dependent on the purpose of the study of the possibility of managing the transport system. According to their purpose among technical systems and relating to the territory, transport systems belong to production systems, including: mining, linear transport, processing and residential complexes.

\section{Results}

The location and ability to manage production systems depends on many conditions - proximity to sources of raw materials, availability of labor resources, energy, water, transport accessibility, etc. Due to the variety of conditions for the location and functioning of transport systems in the general environment of natural and technical systems, the question arises of approaches to their study. General approaches to the study of natural and technical systems were developed by A. Yu. Reteyum. According to his ideas, consideration of the 
interaction of technical and natural systems should be carried out using the following directions [1-9] Dynamic approach. In a dynamic approach, the subject of analysis is the movement of matter, energy and information in a geotechnical environment, mass and energy exchange and the exchange of information between its artificial and natural parts. Moreover, the informational aspect is of paramount importance, since it acquires the meaning of the control action. Particularly important is the detailing of the stage of creating a system and adapting it to the environment. System approach. It is focused on establishing links between the system and its environment and the system's response to changing external conditions. This makes it possible to more fully ascertain the properties of a specific geotechnical system manifested in the interaction of objects with the environment, including an analysis of the spatial relationships of geotechnical systems.

Comparative approach. It is associated with the study of systems by comparing them among themselves and with various interacting geosystems. This approach, which continues the line of the traditional comparative geographical method, makes it possible to clearly identify the specifics of organizing direct interaction or through subsystems with nature and other systems on controlled areas of the earth's surface.

Strategic approach. Strategy and concept are the fundamental concepts of strategic, tactical and operational management, the interconnection of which is shown in figure 1.

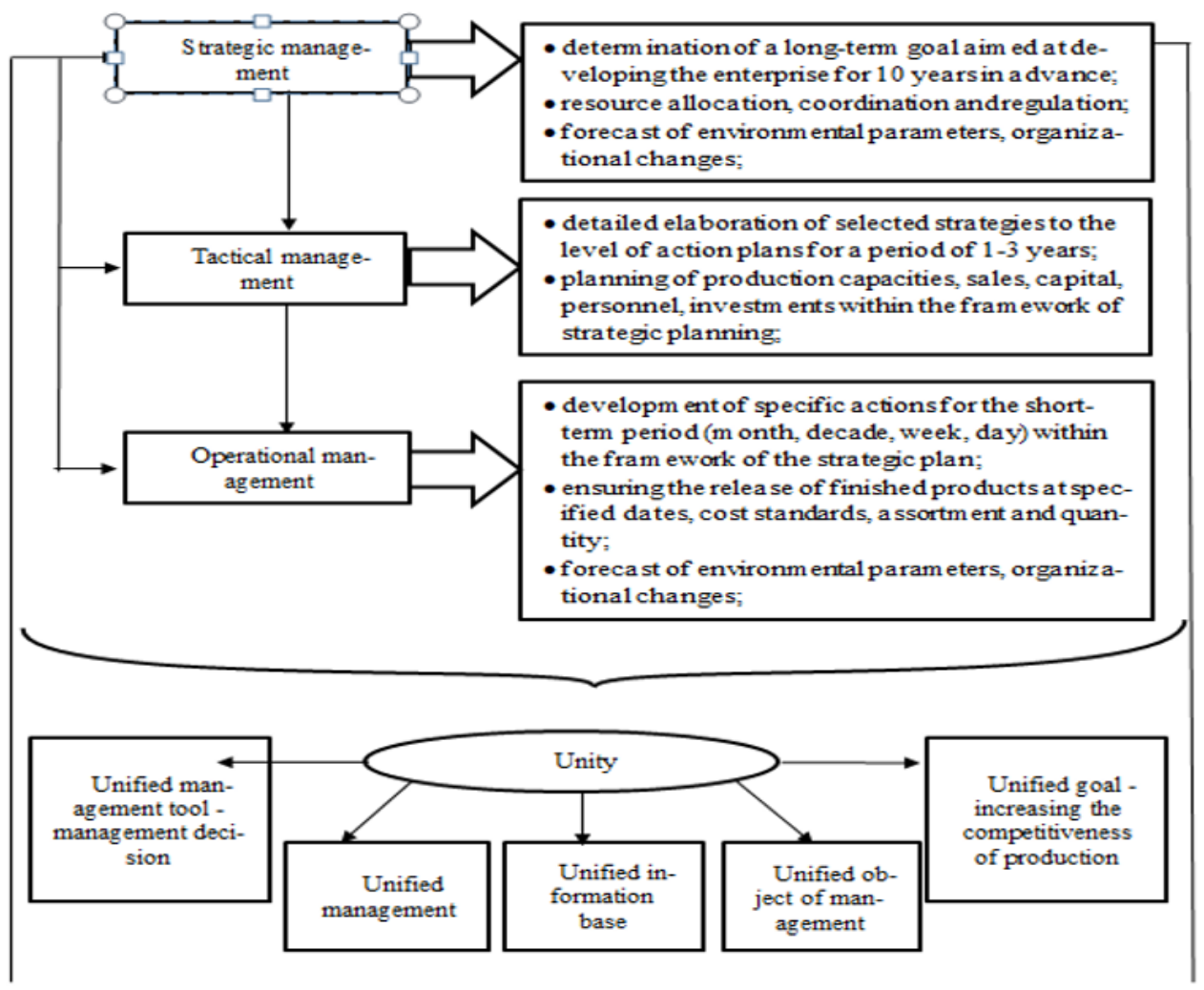

Fig. 1. Relationship between operational, tactical, and strategic management. 
There are models built on the basis of the "Concept-Strategy" principle (Fig.2).

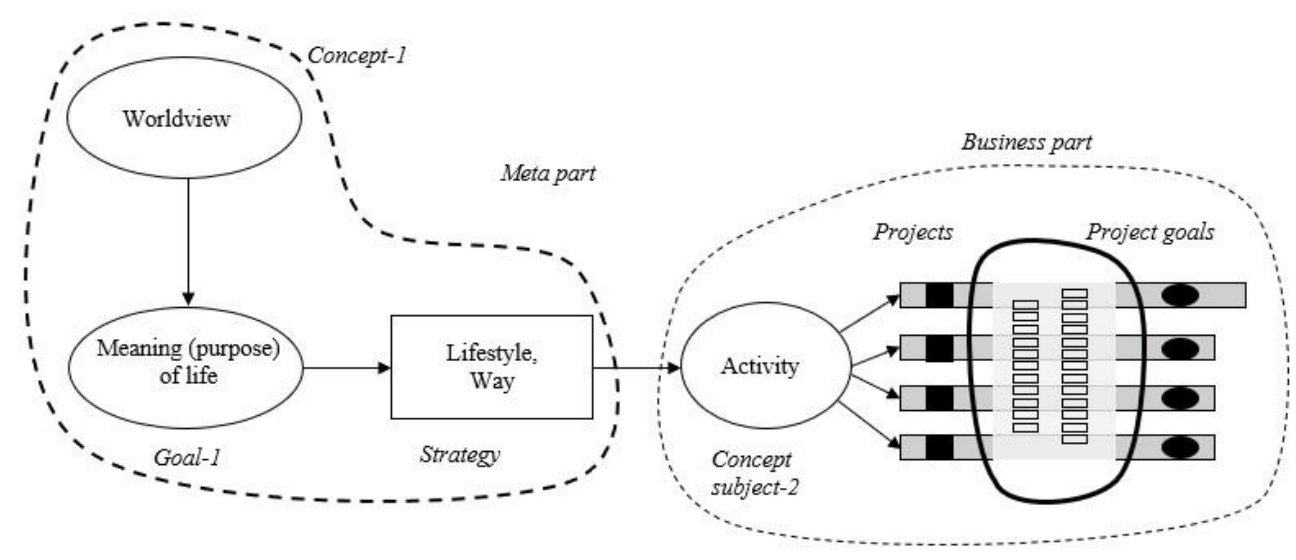

Fig. 2. "Concept - Strategy" model.

The minimum number of experts who must participate in the study to create a representative amount of statistical data, based on a given reliability of the result $(\mathrm{A}=0.95)$, is determined by the formula [10-13].

where

$$
\mathrm{E}=\frac{\mathrm{h}^{2} \cdot \mathrm{r}_{\mathrm{a}} \cdot \mathrm{r}_{\mathrm{o}}}{\Delta^{2}}
$$

$\mathrm{E}$ - minimum necessary number of experts;

$\mathrm{h}$ - confidence coefficient (0.95);

$r_{a}$ - proportion of sample elements with the presence of this attribute $(0.95)$;

$\mathrm{r}_{\mathrm{o}}$ - proportion of sample elements without the presence of this attribute $(0.05)$;

$\Delta-$ error of representativeness $(0.05)$;

$$
\mathrm{E}=\frac{(0.95)^{2} *(0.95) *(0.05)}{(0.05)^{2}}=17.2 \sim 18 \text { persons }
$$

It should be noted that the natural environment is a complex reorganizing and selfregulating system with direct and inverse interconnects. The saturation of the natural environment with engineering objects during the creation of a new natural transport system changes the existing structure of relations and breaks the "chains" of its self-organization, which leads to the occurrence of a new system of higher order of complexity than the original natural environment. The occurrence of such a system logically requires the creation of a control system adequate in terms of its complexity; otherwise, the so-called "von Neumann effect" occurs, i.e. degradation and destruction of the created natural and technical system.

\section{Discussion}

To study the conditions for the creation and development of transport systems of the territories, the complex implementation of all these approaches is needed to obtain versatile information on the state and functioning of the natural and technical systems of the territory under study. This is possible only if there is a formalized model created on the basis of an objective function expressing the dependence of the quality of land use, energy, resources, 
and information on the nature and intensity of functioning in the natural and technical systems of each type of transport.

The study of the natural transport system belongs together with the issues of the development of a integrated transport system of the Russian Federation. For all types of transport, the creation of a transport system and the safety of its interaction with the environment depends on the volume of traffic and the parameters of its protection from the effects of negative processes. This, in general, is traditional not only in Russia, but throughout the world. Ecologically, the safest modes of transport are sea, river and pipeline, despite the fact that the first two are limited to the hydrographic network, and the third - the nature of the transported goods and their different ways of moving (for example, coal, metal, oil, gas).

To ensure mass land freight traffic over long distances, the safest and most efficient is rail transport. This is due to better indicators of energy consumption and lower pollutant emissions per unit of transportation work due to more stable working conditions of diesel engines and the widespread use of electric traction.

For the railway, the environment is other technical systems and nature with which it is in contact. The railway in conjunction with the environment presents a natural and technical system. In the design and operational management of the construction and operation of the railway, the adopted technical, organizational and technological solutions should ensure sustainability at all stages of its life cycle. To this end, it is necessary to create an effective operational management mechanism that would allow continuous monitoring of its parameters and their management, taking into account the current situation in the environment.

The railway can be represented as a complex hierarchy of technical systems, consisting of interconnected and interacting subsystems of different order and elements:

The concepts of system, subsystem and element are relative and are introduced depending on the subject and purpose of the study.

The railway can also be considered as a subsystem included in the Integrated transport system of the country, where other subsystems are road, river, sea, pipeline, air transport.

The organization of usage of each type of transport on a specific territory can be considered as the implementation of the creation of a large serviced natural and technical system "Transport" consisting of two subsystems:

- Stationary - tracks and structures related to the territory and working "to the point of exhaustion" in the perception of the movement of cargo and passenger traffic.

- Movable - trains, locomotives and cars - related to the traffic route and working on the movement of specific goods and passengers.

The subsystems are interconnected, have the structure and control for the operation of information-based and feedback received from their interactions, time between failures, and "recovery" after maintenance (repair, replacement of elements, charging with energy, reconstruction, etc.). Such consideration shows that both subsystems and systems as a whole can relate to cybernetic systems possessing the reliability phenomenon in all three of its implementations: informational, structural, and functional.

Information reliability is realized by surveys and monitoring; structural reliability is realized by design, construction and testing; functional reliability is realized by the safety of the carriage and traffic capacity of the system. 
Both the creation and exploitation (operation) of the system and both subsystems are costly. The expenditure of material, labor and time resources on the creation and operation of subsystems is proportionally different.

The first one has a maximum for creation and for reconstruction and a minimum for operation (repair).

The second one has a maximum during operation; during creation, the attracted resource from other systems of the country's railways network can be used. Both the system as a whole and the subsystems belong to reserved systems.

The first one uses the so-called "cold reserve", a safety factor consumed during operation and requiring replenishment when traffic capacity is exhausted (opening of the secondturn passing loops, construction of the second track).

The second one uses the so-called "hot reserve" - connecting to the work of more powerful rolling stock, attracting rolling stock from neighboring roads, increasing the weight of the train when the carrying capacity is exhausted.

Each of the subsystems, while solving its task, may have its own management, and the system as a whole must have a management coordinating the management of the subsystems, ensuring the optimal participation of each of them in solving the transportation task.

It is assumed that the system as a whole is backed up by logistics, the use of all three implementations of reliability in solving the transportation problem allows connecting it to the control of a specific "transport" system on a specific territory, and the use of logistics is possible by creating new connections of the transport system under consideration with another type of transport, due to the formation of a new transport network in the existing network, and using a part of a different type of transport network, rehandling facilities and terminals. Creating a new connection can also be carried out at the macro level by building the missing part of the network, attracting not one but several types of transport, replacing the delivery of cargo with its production at the place of consumption, i.e. exit from the considered transport system into the production system with the solution of another class of problems. For decades, many scientists, organizations and government structures have been involved in issues and problems of transport development and the design of transport systems. The special contribution to the design of the transport system was made by L.V. Kantorovich. He carried out a general analysis of the transportation problem. One of the variants of the proposed solutions was the method of "potentials". This method is widely used in transport (rail, road, sea, air) and in centralized supply authorities for rational assigning of suppliers to consumers and rational organization of transportation.

Under the concept of "transport problem", a wide range of tasks with a single mathematical model is defined. These tasks are related to linear programming problems.

The transport problem (classical) is the problem of the optimal plan of transportation of a homogeneous product from homogeneous points of their presence to homogeneous points of their consumption on homogeneous vehicles (in a predetermined quantity) with static data and a linear approach. These are the basic conditions of the task, which are also constraints, since homogeneity excludes the dynamics of the management process.

For the classical transport problem, when searching for the optimal solution, two types of goals are distinguished: by the criterion of cost (achieving the minimum cost of transportation) or distances, and by the criterion of time (the minimum of time is spent on transportation).

Of the previously developed three methods for solving the transport problem (the "north - west angle" method, the "tariffs" method, and the "potentials" method), the "potentials" method retains its value along with the currently widely used method of dispatch control and the design route model. The method was investigated by L.V. Kantorovich and M.K. Gavrushin in 1940, and it was finally formed by L.V. Kantorovich in 1949. 
The issues of an integrated transport system: the interrelation of transport with other sectors of the national economy, the distribution of traffic between two types of transport, taking into account economy and, in particular, energy, remain relevant today.

However, the transport problem in all three statements of its solution is considered under the conditions of a transport network connecting production points and a consumption point with varying possible redundancy of "stock" or "demand" for cargo transportation.

The formation of a local logistics network, which is based on methodological principles and methodological approaches tested on new built transport construction facilities, is important. A properly set logistics system ensures the management of the timeliness of resource supplies, which is one of the factors for meeting the contractual deadlines.

The possibility of varying the design of a network of several types of transport and building new connections with the involvement of logistics, as well as the possibility of varying the quality of the existing network - reconstruction, innovation in construction and operation, gives rise to a new type (and possibly class) of tasks whose solution is necessary for the rational development of an integrated transport system (ITS) of individual regions [5-7] and the country as a whole. From this point of view, the source for developing a mathematical framework can be the imparting of "potentials" with the probability of logistic links, which makes it possible to attract the reliability theory for minimizing risks in forecasting, planning, designing and building transportation networks to create the development and management of an integrated transport system in its most costly part - the complex of stationary subsystems of transport $[4,6]$.

It should be expected that with rational management of construction production in the course of creating new connections and reconstruction of the existing transport network facilities in the life cycle of stationary subsystems of land types of transport in the Integrated Transport System, a significant effect can be achieved both at the initial stage of the ITS formation and during the implementation of construction projects.

\section{Conclusions}

Sound management allows quickly solving a whole range of problems arising during construction, and most importantly, save time by responding to changes in the parameters and conditions of the project; estimating the critical path and determining the real time reserves of the work; performing cost analysis in time sections; creating logistic schemes for the implementation of various project scenarios and the search for effective technologies by regulating the composition of teams, the type of materials, and the means of mechanization used; simulating risks, calculating realistic terms and budgets of projects, determining the necessary reserves, the possibilities for introducing innovations, taking into account trends and tracking the likelihood of their changes.

In order to implement the methodology for managing projects for the construction of transport facilities, principles have been developed for developing individual project management algorithms, including organizational preparatory procedures, which should be mandatory for all project participants, including customers. This is especially important for saving labor resources and time as a resource. The experience of implementing the developed methodology [1-3] showed the possibility of creating a management system for PM, which allows the project information to be used by all its participants, improving efficiency 
and determining the direction of improving organizational and technological solutions through operational management of the resulting deviations, shortening the construction time of objects, rational use of resources, reducing the development time of projects of unique objects, ensuring the possibility of conducting internal and external monitoring and audit. The use of the developed methodology in the construction of a bridge overpass over the river Volga in Dubna allowed reducing the duration of the construction project by 101 calendar days (from 660 days to 559 days); receiving financial savings of $16 \%$ of the initial cost, improving the technology of work production, and more than five times reducing labor intensity.

\section{References}

1. A.A.Volkov, V.O.Chulkov, R.R.Kazaryan, S.A.Sinenko, Applied Mechanics and Materials 584-586, 2681-2684 (2014). Doi: 10.4028/www.scientific.net/AMM.584586.2681 .

2. S.A. Sinenko, A.O. Feldman, IOP Conf. Series: Materials Science and Engineering, 463, (2018). doi:10.1088/1757899X/463/4/042010.

3. K. Yu. Losev, V. O. Chulkov, R.R. Kazaryan, IOP Conf. Series: Materials Science and Engineering, 463, (2018)

4. Z. Jakšic, D. Ladjinović, M. Trivunić, N. Harmati, N. Vatin. Procedia Engineering. 2015. 117(1). Pp. 502-515. DOI:10.1016/j.proeng.2015.08.252.

5. T.V. Nazmeeva, N.I. Vatin. Magazine of Civil Engineering 62(2). 92-101. (2106) DOI:10.5862/MCE.62.9.

6. Ulybin, A. Lanko, N. Vatin, K. Lysnytska. MATEC Web of Conferences. 73. (2106) DOI:10.1051/matecconf/20167301007. 